\title{
Discussion: Use of viscous pore fluids in dynamic centrifuge modelling
}

Carlos Lam DPhil, CEng, MICE, MHKIE

Geotechnical Engineer, Geotechnical Engineering Office, Civil Engineering and Development Department, The Government of the Hong Kong Special Administrative Region, Hong Kong; Formerly Lecturer in Geotechnical Engineering, School of Mechanical, Aerospace and Civil Engineering,

The University of Manchester, Manchester, UK
John O. Ejezie BEng, MSC

Postgraduate Student, School of Mechanical, Aerospace and Civil Engineering, The University of Manchester, Manchester, UK

Stephan A. Jefferis PhD, CEng, CEnv, FICE, CGeol, FGS, FRSA Director, Environmental Geotechnics Ltd, Adderbury, Banbury, UK; Visiting Professor, Department of Engineering Science, University of Oxford, Oxford, UK

\section{Contribution by C. Lam, J.O. Ejezie and S.A. Jefferis}

Adamidis and Madabhushi (2015) presented interesting results of viscosity determinations on hydroxypropyl methylcellulose (HPMC) solutions that have been used as a viscous pore fluid in dynamic centrifuge modelling. The effects of concentration, temperature, ageing and shear rate on viscosity were studied. This discussion raises a few points in relation to the effect of polymer solution concentration and identifies some other factors that can affect solution viscosity.

On the basis of the test results, the authors proposed a simple power-law relationship (Equation 6) to fit the viscosity results over a range of polymer concentrations, as follows

6. $\mu=8 \cdot 017 c^{2 \cdot 715}$

where $\mu$ is the apparent viscosity in $\mathrm{mPa} \mathrm{s}$ and $c$ is the concentration in per cent by weight. However, this equation has the shortcoming that the predicted viscosity will be zero if the polymer concentration is zero, whereas at zero concentration the viscosity value should be that of water, that is, $1.0 \mathrm{mPa}$ s at $20^{\circ} \mathrm{C}$. Equation 6 can be easily modified to include an additional term to account for water viscosity, as shown in Lam et al. (2015), to obtain the viscosity-concentration relationships for partially hydrolysed polyacrylamide solutions, as follows

$$
\mu=1+A c^{B}
$$

where $A$ and $B$ are constants depending on the physiochemical properties of the polymer and the governing shear rate. Note that the Greek letter ' $\eta$ ' instead of ' $\mu$ ' was used in Lam et al. (2015) to denote viscosity; however, both are equally common.
On the basis of the same set of viscosity data and using an equation similar to that given in Dow (2002), the authors also propose that centrifuge modellers could adopt the following equation if no viscosity measurement apparatus is available

7. $\mu=(0 \cdot 268 c+1.094)^{8}$

Again, this equation has the shortcoming that at zero concentration the predicted viscosity will not be that of water; in this case, predicted viscosity is $2 \cdot 05 \mathrm{mPa} \mathrm{s}\left(=1 \cdot 094^{8}\right)$. A review of Dow (2002) reveals that their equation actually has the following form

$$
\mu^{1 / 8}=\alpha c+1
$$

where $\alpha$ is a constant specific to the molecular weight (or viscosity grade) of the polymer and the constant 1 is the viscosity of water at room temperature. It is important to accurately represent low concentrations as HPMC solutions may be required with viscosities as low as $10 \mathrm{cSt}$ (equivalent to $10 \mathrm{mPa}$ s when fluid density is $1 \mathrm{~g} / \mathrm{cm}^{3}$ ) for centrifuge modelling according to Stewart et al. (1998).

When discussing the viscosity data presented in their Figures 2 and 3 , the authors noted that the difference in results as compared with those of Stewart et al. (1998) could have been due to the particular properties of the polymers being tested. However, the viscosity results given in Stewart et al. (1998) were obtained using several Ubbelohde-type capillary viscometers (see e.g. ASTM D 2363 (ASTM, 2011)), whereas the authors had used a Brookfield rotational viscometer (model DV-I+). It is important to recognise that if the fluid being assessed is non-Newtonian, as HPMCs are, then viscosity data generated using different techniques will be comparable 
Discussion

Lam, Ejezie and Jefferis only if the governing shear rates for the respective tests are comparable. It is to be noted that the shear rates used for the authors' measurements are not given.

A further point in relation to shear rates is that the authors' viscosity measurements were made over a range of shear rates and an average value was used. The use of an average shear rate is not advised, for as shown in Figure 7, HPMC solutions exhibit non-Newtonian behaviour. The use of an average viscosity may explain the slightly different results the authors obtained for repeated measurements (Figures 2 and 3). It is suggested that a better approach would be to use a single shear rate throughout the test programme, except for any necessary study of the effect of different shear rates. The value of shear rate used should be appropriate to the geotechnical problem being studied. It should also be noted that, in addition to the effect of shear rate, there are a number of other significant influences on the viscosity of polymer solutions, including the following.

- Polymers can be sorbed on to soils (Lam et al., 2014). This will reduce the active concentration of polymer in the soil pores and hence, the pore fluid viscosity. Sorption also may reduce pore throat diameters. Effects will be most significant in fine soils of high specific surface area.

- Polymer solution viscosity can be markedly reduced by salts in solution (Jefferis and Lam, 2013). It is important that solutions are prepared with distilled or deionised water, and that soils are washed to remove dissolved species prior to use. If soils are to be re-used, these should be washed free of all residual polymer prior to the re-use.

- While the authors have cited polymer-coated pills as an example of resistance to biodegradation, it should be remembered that pills will be ineffective unless at some stage in the passage through the digestive system the coating is lost and the content released. Although initial rates of biodegradation may be low, once infection has occurred and a sufficient colony of degraders has developed, degradation can be very rapid. Therefore, soils should be sterilised prior to use or re-use; to this end, simple oven drying after washing with pure water may suffice.

\section{Authors' reply}

The authors thank the discussers for closely reading the paper (Adamidis and Madabhushi, 2015) and for raising some important points. The issues that have been mentioned will be addressed in the following paragraphs.

The discussers pointed out that the curve-fits proposed in Equations 6 and 7 for viscosity as a function of HPMC concentration do not capture the viscosity of water at zero concentration of HPMC. The authors are in agreement about the inaccuracy at zero concentration. Further, since these expressions are curve-fits, the authors suggest that these should not be extrapolated to any value outside of the range of the measurements on which these were based. As stated in the original paper, the expressions of Equations 6 and 7 are best fits for the measurements presented in Figures 2 and 3, where HPMC concentrations range from $1 \cdot 4$ to $3 \cdot 2 \%$, and viscosities from about 20 to $200 \mathrm{mPa}$ s. Indeed, the plots in Figures 2 and 3 were not continued for concentrations lower or higher than the measurements. The choice of these expressions reflects the purpose of the authors' work that was to give a practical guideline for the preparation of high-viscosity pore fluids for dynamic centrifuge tests. A realistic range of $\boldsymbol{g}$-levels for such tests would be from 30 to $100 \mathrm{~g}$, requiring pore fluids with viscosities from 30 to $100 \mathrm{mPa}$. The authors chose to examine a range of viscosities from 20 to $200 \mathrm{mPa}$ s that were expected to cover the needs of dynamic centrifuge testing. The proposed expressions are meant to be used within this range of viscosity. Lower or higher concentrations and viscosities, therefore, were not considered.

The power law of Equation 6 was

$$
\mu(\mathrm{mPa} \mathrm{s})=8.017 c(\%)^{2.715}, \quad R^{2}=0.9917
$$

where $\mu$ signifies dynamic viscosity in $\mathrm{mPa}$ s and $c$ signifies the fraction of the mass of HPMC over the mass of water, in percentage. The $R^{2}$ value corresponds to how good the fit is for the measurements shown in Figures 2 and 3 of the original paper. If the type of equation proposed by the discussers was used (Lam et al., 2015), the expression would be as follows

$$
\mu(\mathrm{mPa})=1+7 \cdot 548 c(\%)^{2 \cdot 768}, \quad R^{2}=0.9917
$$

The fits are equally good for the range of interest. For a solution of $20 \mathrm{mPa} \mathrm{s}$, at the lower edge of viscosities considered, the difference in required concentrations - as calculated with the two expressions - is $<0 \cdot 3 \%$. The authors opted for the simpler expression.

With regard to the expression represented by Equation 7, the eighth power rule proposed by the authors was

$$
\mu(\mathrm{mPas})=(0 \cdot 268 c(\%)+1.094)^{8}, \quad R^{2}=0.9945
$$

If a constraint is introduced so that the viscosity of water at zero concentration is captured, the expression becomes

$$
\mu(\mathrm{mPa} \mathrm{s})=(0 \cdot 309 c(\%)+1)^{8}, \quad R^{2}=0.9703
$$


Discussion

Lam, Ejezie and Jefferis

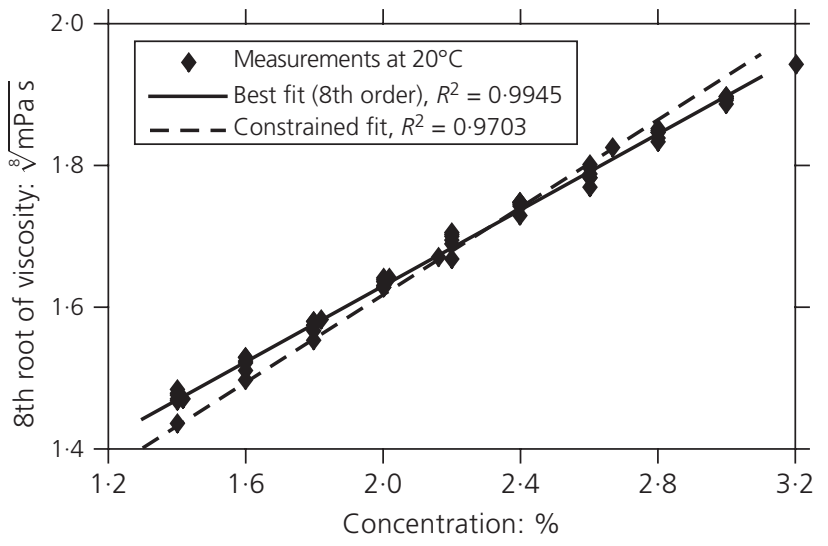

Figure 1. Eighth root of viscosity change with concentration. The constrained fit captures the viscosity of water at zero concentration but offers a worse fit for the range of interest

The two expressions are plotted along with the measurements in Figure 1. The second expression provides a worse fit for the data. The authors chose to propose the use of the expression that most accurately captures viscosity for the range of interest.

The discussers also pointed out that since the HPMC solutions were non-Newtonian (Figure 7 of the original paper), the measured viscosity depends on the shearing rate of the measurement. Therefore, the use of an average viscosity, taken from measurements with different shear rates, was stated as not advisable. The authors agree with the discussers and regret not including the shearing rates of the measurements in the original paper, as this point could then have been clarified. Three viscosity measurements were made for each sample using the UL adapter of a Brookfield LV DV-I+ viscometer. The following three shearing rates were used: $0 \cdot 37,0.73$ and $1.83 \mathrm{~s}^{-1}$. In this range of shearing rates, HPMC solutions in the viscosity range when examined practically behave as Newtonian fluids (Figure 7 of the original paper). Consequently, viscosity was practically constant over the three shearing rates of the measurements, justifying the use of an average value. As mentioned in the original paper, variations in viscosity at different shearing rates were small and remained in all cases below $4 \mathrm{mPa}$ s. If only one of the three shearing rates was used, as suggested by the discussers, the results would remain practically unchanged. Moreover, the shearing rates used were also within the expected range of shearing rates for dynamic centrifuge tests (shaded area of Figure 7 of the original paper). Therefore, the expressions presented for viscosity by the authors should be expected to be accurate for dynamic centrifuge testing applications.

It is worth mentioning that identifying a single important shearing rate for a dynamic centrifuge test can be difficult, as shearing rates can change significantly along with the velocity of pore fluid. For instance, in the case of a liquefiable sand layer, the whole range of shearing rates shaded in Figure 7 of the original paper would be covered. In such cases, it is important to know if within the expected range of shearing rates, the change in viscosity is small enough for the pore fluid to be assumed to behave as a Newtonian fluid and if so, what the average value of viscosity will be.

The discussers listed some further factors that can affect viscosity that were not considered by the authors.

First, it was pointed out that sorption is a mechanism that can reduce the concentration of polymer and hence, the viscosity of pore fluids. The authors did not study sorption as it was not considered important for dynamic centrifuge testing. Highviscosity pore fluids are typically employed for models that test sand, whose low specific area limits the effects of sorption. Indeed, the viscosity of pore fluids is routinely measured before and after dynamic centrifuge tests performed at the University of Cambridge and no significant change has been observed. This suggests that sorption effects are not significant for the time scale and the type of sand typically used in dynamic centrifuge tests.

Second, the issue of the effect of salt concentration on viscosity was raised (Jefferis and Lam, 2013). The authors agree that distilled or deionised water should be used for the preparation of polymer solutions. The samples examined by the authors were prepared with deaired, deionised water, and the effect of salt concentration was not studied further.

Afterwards, possible biodegradation of HPMC solutions was addressed. The discussers proposed sterilisation by washing and oven drying of the soil. Since this process can become impractical for large quantities of soil and does not shield the model from future infection, centrifuge modellers often use chemicals for sterilisation. The authors examined the ageing of samples for a month and did not notice any degradation effect, as reported in the original paper. Models prepared for dynamic centrifuge tests only remain saturated for a few days, since deaired pore fluids are used. In the authors' experience, within this time frame, the inherent resistance of HPMC solutions to biodegration is sufficient to maintain a constant viscosity and sterilisation is not necessary. When using chemicals, their effect on viscosity should be examined beforehand.

Finally, the authors agree with the discussers on the points they raise about the re-use of sands that have already been saturated with HPMC solutions for a previous test. To the authors' knowledge, worldwide practice is that sands used for dynamic saturated centrifuge tests are discarded and fresh, dry 
sand is used to prepare new models. The authors are not aware of any dynamic centrifuge tests where sand saturated with a high-viscosity fluid is dried and re-used.

\section{REFERENCES}

Adamidis O and Madabhushi GSP (2015) Use of viscous pore fluids in dynamic centrifuge modelling. International Journal of Physical Modelling in Geotechnics 15(3): 141-149, http://dx.doi.org/10.1680/jphmg.14.00022. ASTM (2011) D 2363-79: Standard test methods for hydroxypropyl methylcellulose. ASTM International, West Conshohocken, PA, USA.

Dow (2002) Methocel Cellulose Ethers: Technical Handbook. The Dow Chemical Company, Staines, UK.
Jefferis SA and Lam C (2013) Polymer support fluids: use and misuse of innovative fluids in geotechnical works. Proceedings of the 18th International Conference on Soil Mechanics and Geotechnical Engineering, Paris, France. Presses des Ponts, Paris, France, vol. 4, pp. 3219-3222.

Lam C, Martin PJ, Jefferis SA and Goodhue KG Jr (2014) Determination of residual concentration of active polymer in a polymeric support fluid. Geotechnical Testing Journal 37(1): 46-59.

Lam C, Martin PJ and Jefferis SA (2015) Rheological properties of PHPA polymer support fluids. Journal of Materials in Civil Engineering 27(11): 04015021.

Stewart D, Chen Y and Kutter B (1998) Experience with the use of methylcellulose as a viscous pore fluid in centrifuge models. Geotechnical Testing Journal 21(4): 365-369. 